\title{
AN EXAMPLE OF NON-COTORSION SELMER GROUP
}

\author{
KING FAI LAI, IGNAZIO LONGHI, KI-SENG TAN, AND FABIEN TRIHAN
}

(Communicated by Matthew A. Papanikolas)

\begin{abstract}
Let $A / K$ be an elliptic curve over a global field of characteristic $p>0$. We provide an example where the Pontrjagin dual of the Selmer group of $A$ over a $\Gamma:=\mathbb{Z}_{p}$-extension $L / K$ is not a torsion $\mathbb{Z}_{p}[[\Gamma]]$-module and show that the Iwasawa Main Conjecture for $A / L$ holds nevertheless.
\end{abstract}

\section{Setting}

Let $K$ be a global field of characteristic $p>0$ and let $A / K$ be a non-isotrivial semistable elliptic curve of analytic rank 0 with split multiplicative reduction at a place $v_{0}$ of $K$. Let $L / K$ be a $\Gamma=\mathbb{Z}_{p}$-extension and $k \subset K$ be a subfield such that $K / k$ is a separable quadratic extension with $\operatorname{Gal}(K / k)=\{1, \tau\}$. We denote for any $n, K_{n} / K$ the $\mathbb{Z} / p^{n}$-extension and set $\Gamma^{(n)}:=\operatorname{Gal}\left(L / K_{n}\right)$ and $\Gamma_{n}=\operatorname{Gal}\left(K_{n} / K\right)$. We assume that $L / K$ is anticyclotomic with respect to $k$, i.e. $L / k$ is Galois and $\operatorname{Gal}(L / k)$ is dihedral in the sense that we have a decomposition

$$
\operatorname{Gal}(L / k) \simeq \operatorname{Gal}(L / K) \rtimes \operatorname{Gal}(K / k)
$$

such that for any $\sigma \in \operatorname{Gal}(L / K)$ we have $\tau \sigma \tau^{-1}=\sigma^{-1}$ (where $\tau$ is abusively identified with its lift to $\operatorname{Gal}(L / k))$.

Let us assume moreover that $L / K$ is totally ramified above $v_{0}$ and unramified elsewhere. Furthermore, $A$ is assumed to be already defined over $k$ and to have split multiplicative reduction at the restriction of $v_{0}$ to $k$.

Remark 1.0.1. There are many known instances of elliptic curves satisfying these hypotheses. For example, let $\mathbb{F}$ be a finite field of characteristic $p>2$ and consider the function fields $k=\mathbb{F}(s)$ and $K=\mathbb{F}(t)$, with $s=t^{2}$. Let $A$ be defined by the Weierstrass equation

$$
A: y^{2}=x(x+1)(x+s)=x(x+1)\left(x+t^{2}\right) .
$$

This is an elliptic curve having split multiplicative reduction at $t=0, t=\infty$ and (if -1 is a square in $\mathbb{F}$ ) $t= \pm 1$, and good reduction at all other places. Therefore, as a divisor of $K$ the conductor $\mathfrak{n}$ of $A / K$ is the sum of these four places. The elliptic curve $A / K$ is well known to have Hasse-Weil L-function $L(A / K, s) \equiv 1$ : one way to prove it is to observe that $A$ is associated with a modular form and apply the corollary of [Tan93, Proposition 3]; another approach (closer to the methods of Ta66]) is explained in Shi92].

Received by the editors August 6, 2013 and, in revised form, January 21, 2014.

2010 Mathematics Subject Classification. Primary 11S40; Secondary 11R23, 11R34, 11R42, 11R58, 11G05, 11G10.

Key words and phrases. Abelian variety, Selmer group, Frobenius, Iwasawa theory, Stickelberger element, syntomic. 
Equation (11) is an instance of Beauville surface: they exist in all characteristics and always satisfy the hypotheses. See Lan91 for a full discussion and classification.

1.1. Let $i: A_{p^{n}} \hookrightarrow A$ be the group scheme of $p^{n}$-torsion of $A$. The $p^{n}$-Selmer group $\operatorname{Sel}_{p^{n}}(A / K)$ is defined to be the kernel of the composition

$$
\mathrm{H}_{\mathrm{fl}}^{1}\left(K, A_{p^{n}}\right) \stackrel{i^{*}}{\longrightarrow} \mathrm{H}_{\mathrm{fl}}^{1}(K, A) \stackrel{l o c_{K}}{\longrightarrow} \bigoplus_{v} \mathrm{H}_{\mathrm{fl}}^{1}\left(K_{v}, A\right),
$$

where $\mathrm{H}_{\mathrm{fl}}^{\bullet}$ denotes the flat cohomology and $l o c_{K}$ is the localization map to the direct sum of local cohomology groups over all places of $K$. The same definition works over any finite extension $F / K$. Taking the direct limit as $n \rightarrow \infty$, we get

$$
\operatorname{Sel}_{p \infty}(A / F):=\operatorname{Ker}\left(\mathrm{H}_{\mathrm{fl}}^{1}\left(F, A_{p} \infty\right) \longrightarrow \bigoplus_{\text {all } v} \mathrm{H}_{\mathrm{fl}}^{1}\left(F_{v}, A\right)\right)
$$

where $A_{p} \infty$ is the $p$-divisible group associated with $A$. The Selmer group $\operatorname{Sel}_{p} \infty(A / L)$ is then defined by taking the inductive limit over all finite subextensions. The Galois group $\Gamma$ acts on $\operatorname{Sel}_{p}(A / L)$ turning it into a $\Lambda:=\mathbb{Z}_{p}[[\Gamma]]$-module. We denote

$$
X_{p}(A / L):=\operatorname{Sel}_{p \infty}(A / L)^{\vee}
$$

the Pontrjagin dual of this $\Lambda$-module.

Similarly, we define for any finite extension $F / K$ the Tate-Shafarevich group of $A / F$ as

$$
\amalg(A / F):=\operatorname{Ker}\left(\mathrm{H}_{\mathrm{fl}}^{1}(F, A) \longrightarrow \bigoplus_{\text {all } v} \mathrm{H}_{\mathrm{fl}}^{1}\left(F_{v}, A\right)\right)
$$

and $\amalg(A / L)$ as the inductive limit of $\amalg(A / F)$ over all finite intermediate extensions.

In this short note, we study the Iwasawa theory of $A / L$ as formulated by Maz72]. According to the Iwasawa Main Conjecture (see [SU13] for the best result so far in the number field case), the characteristic ideal of the finitely generated $\Lambda$-module $X_{p}(A / L)$ should coincide with the ideal generated by an element of $\Lambda$ called the $p$-adic $L$-function and having the property to interpolate the values at $s=1$ of the Hasse-Weil $L$-function of $A / K$ twisted by characters. Here we provide under our assumptions on $A$ and $L / K$, a proof of the Iwasawa Main Conjecture in the following sense: we show in $₫ 2$ that $X_{p}(A / L)$ is a finitely generated non-torsion $\Lambda$ module with therefore a trivial characteristic ideal. In $\$ 3$, we show that the $p$-adic $L$-function of $A / L$ is zero proving as a consequence the Iwasawa Main Conjecture for $A / L$.

In the following, with a slight abuse of notation we shall often use the same symbol to denote places in different fields: e.g., $L_{v_{0}}$ will be the completion of $L$ at the only place above $v_{0}$. Also, for $v$ a place of $K$ put $\Gamma_{v}:=\operatorname{Gal}\left(L_{v} / K_{v}\right)$. Since $\Gamma_{v_{0}}$ can be identified with $\Gamma$, we won't distinguish between the two. Various restriction maps will be used. Among them, $\operatorname{Res}_{L / K_{n}}$ and $\operatorname{res}_{L / K_{n}}$ are respectively the maps $\mathrm{H}_{\mathrm{fl}}^{1}\left(K_{n}, A_{p^{\infty}}\right) \rightarrow \mathrm{H}_{\mathrm{fl}}^{1}\left(L, A_{p^{\infty}}\right)^{\Gamma^{(n)}}$ and $\mathrm{H}_{\mathrm{fl}}^{1}\left(K_{n}, A\right) \rightarrow \mathrm{H}_{\mathrm{fl}}^{1}(L, A)^{\Gamma^{(n)}}$.

\section{The ALGEBRAIC SIDE}

In the following, we fix a topological generator $\gamma$ of $\Gamma$ and put $T:=\gamma-1 \in \Lambda$; then $T$ is a generator of the augmentation ideal $I$. By abuse of notation, we also identify $\gamma$ with a generator of $\Gamma_{n}$ for all $n$. 
Lemma 2.0.1. The groups $A(K)$, $(A / K)$ and $\operatorname{Sel}_{p^{\infty}}(A / K)$ are all finite.

Proof. By [Ta66] (and Mil75] for the p-part of $\amalg$ - see also the comments on Milne's webpage http://www.jmilne.org/math/articles/index.html for $p=2$ ) it is known that analytic rank 0 implies that the full Birch and Swinnerton-Dyer conjecture holds for $A / K$.

Since $A$ has split multiplicative reduction at $v_{0}$, it is a Tate curve on the completion $K_{v_{0}}$ : we denote the local Tate period by $Q$.

Lemma 2.0.2. One has $A_{\text {tor }}(K)=A_{\text {tor }}(L)$. In particular, the group $A_{\text {tor }}(L)$ is finite.

Proof. To see this, first notice that the constant field does not grow in $L_{v_{0}} / K_{v_{0}}$, since it is a totally ramified extension. Besides, if a root $Q^{1 / n}$ of $Q$ is not already in $K_{v_{0}}$, then it cannot belong to $L_{v_{0}}$, either because then $Q^{1 / n}$ is not separable over $K_{v_{0}}$ (for $n$ a power of $p$ ) or because $L / K$ is a $p$-extension (for $(p, n)=1$ ). The explicit description of the torsion points of $A\left(L_{v_{0}}\right)$ in terms of roots of unity and of $Q$ then implies $A_{t o r}\left(K_{v_{0}}\right)=A_{\text {tor }}\left(L_{v_{0}}\right)$. To conclude, it suffices to observe that $L \cap K_{v_{0}}=K$, exploiting once again the totally ramified hypothesis.

Corollary 2.0.3. The groups $\mathrm{H}^{1}\left(\Gamma^{(m)}, A_{p}(L)\right)$ are finite of bounded orders.

Proof. By the usual Herbrad quotient argument, for every $n \geq m$,

$$
\left|\mathrm{H}^{1}\left(K_{n} / K_{m}, A_{p^{\infty}}\left(K_{n}\right)\right)\right|=\left|\hat{\mathrm{H}}^{0}\left(K_{n} / K_{m}, A_{p^{\infty}}\left(K_{n}\right)\right)\right| \leq\left|A_{p^{\infty}}\left(K_{m}\right)\right| \leq\left|A_{p^{\infty}}(L)\right| .
$$

2.1. If $v$ is in the $\operatorname{Gal}(K / k)$-orbit of $v_{0}$, then since $L / k$ is Galois, $v$ is ramified under $L / K$, and hence by our ramification hypotheses, $v=v_{0}$. Besides, we are assuming that $A$ is already a Tate curve over $k_{v_{0}}$ and thus we have $Q \in k_{v_{0}}^{\times}$.

Let $\mathcal{N} \subseteq K_{v_{0}}^{\times}$denote the group of universal norms of the local extension $L_{v_{0}} / K_{v_{0}}$. Write rec for the reciprocity map of local class field theory: then we have $\operatorname{rec}(\tau(x))=$ $\tau \operatorname{rec}(x) \tau^{-1}$ for any $x \in K_{v_{0}}^{\times}$, and hence $\tau(x) \equiv x^{-1} \bmod \mathcal{N}$. In particular $Q \equiv Q^{-1}$ $\bmod \mathcal{N}$, so that $Q^{2} \in \mathcal{N}$. As $K_{v_{0}}^{\times} / \mathcal{N} \simeq \Gamma$ is torsion free, we deduce that $Q \in \mathcal{N}$.

Lemma 2.1.1. Let $v$ be a place of $K$. Then

$$
\mathrm{H}^{1}\left(\Gamma_{v}, A\left(L_{v}\right)\right) \simeq \begin{cases}0 & \text { if } v \text { is a place of good reduction; } \\ \text { a finite group } & \text { if } v \text { is an unramified place of bad reduction; } \\ \mathbb{Q}_{p} / \mathbb{Z}_{p} & \text { if } v=v_{0} .\end{cases}
$$

Proof. For unramified places, this is a consequence of Mil86a, I, Proposition 3.8]. As for $v_{0}$, observe that for any $n$ we have $A\left(K_{n, v_{0}}\right) \simeq K_{n, v_{0}}^{\times} / Q^{\mathbb{Z}}$. We deduce the exact sequence

$$
\mathrm{H}^{1}\left(\Gamma_{n}, K_{n, v_{0}}^{\times}\right) \longrightarrow \mathrm{H}^{1}\left(\Gamma_{n}, A\left(K_{n, v_{0}}\right)\right) \longrightarrow \mathrm{H}^{2}\left(\Gamma_{n}, Q^{\mathbb{Z}}\right) \longrightarrow \mathrm{H}^{2}\left(\Gamma_{n}, K_{n, v_{0}}^{\times}\right),
$$

that we can rewrite

$$
0 \longrightarrow \mathrm{H}^{1}\left(\Gamma_{n}, A\left(K_{n, v_{0}}\right)\right) \longrightarrow Q^{\mathbb{Z}} / Q^{p^{n} \mathbb{Z}} \longrightarrow K_{v_{0}}^{\times} / N_{K_{n, v_{0}} / K_{v_{0}}}\left(K_{n, v_{0}}^{\times}\right) .
$$

Since $Q \in \mathcal{N}$, the map $Q^{\mathbb{Z}} \rightarrow K_{v_{0}}^{\times} / N_{K_{n, v_{0}} / K_{v_{0}}}\left(K_{n, v_{0}}^{\times}\right)$is trivial. Thus we obtain

$$
\mathrm{H}^{1}\left(\Gamma_{n}, A\left(K_{n, v_{0}}\right)\right) \simeq p^{-n} \mathbb{Z} / \mathbb{Z} .
$$


As $n$ varies, this isomorphism is compatible with the inflation maps on the left and the canonical inclusions on the right, thereby proving the assertion.

Corollary 2.1.2. The group $\operatorname{Sel}_{p} \infty(A / L)^{\Gamma}$ is cofinitely generated over $\mathbb{Z}_{p}$ of corank at most one. The $\Lambda$-module $X_{L}$ is finitely generated.

Proof. Applying the snake lemma and the Hochschild-Serre spectral sequence (Mil80, III, 2.21]) to the diagram

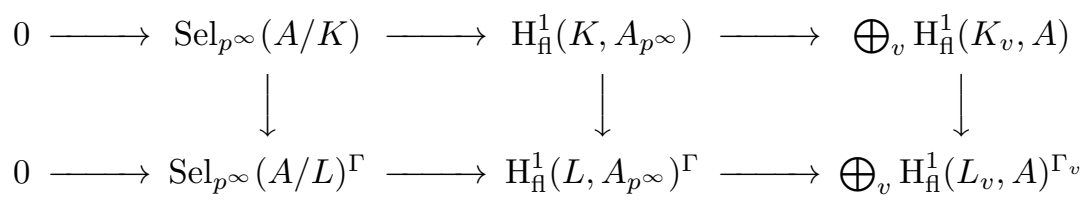

we get an exact sequence

$$
\mathrm{H}^{1}\left(\Gamma, A_{p^{\infty}}(L)\right) \longrightarrow H \longrightarrow \operatorname{Sel}_{p^{\infty}}(A / L)^{\Gamma} / \operatorname{Sel}_{p^{\infty}}(A / K) \longrightarrow \mathrm{H}^{2}\left(\Gamma, A_{p^{\infty}}(L)\right)=0,
$$

where $H$ is a subgroup of $\oplus_{v} \mathrm{H}^{1}\left(\Gamma_{v}, A\left(L_{v}\right)\right)$ and the equality on the right comes from the fact that $\Gamma$ has cohomological dimension 1 . The claim follows from Lemma 2.1.1, since $A_{p \infty}(L)$ and $\operatorname{Sel}_{p \infty}(A / K)$ are finite groups thanks to Lemmas 2.0.2 and 2.0.1. The second assertion is a consequence of the first by the usual topological Nakayama Lemma.

Remark 2.1.3. The group $G:=\operatorname{Gal}(K / k)$ acts on $\mathrm{H}_{\mathrm{fl}}^{1}(K, A)$ and the other cohomology groups appearing in the above discussion. In particular, since $v_{0}$ does not split in $K / k, G$ acts on $\mathrm{H}^{1}\left(\Gamma, A\left(L_{v_{0}}\right)\right)$. The connecting homomorphism in (5) is a map of $G$-modules and so is the isomorphism (6), because $Q \in k_{v}$. Therefore we have

$$
\mathrm{H}^{1}\left(\Gamma, A\left(L_{v_{0}}\right)\right)=\mathrm{H}^{1}\left(\Gamma, A\left(L_{v_{0}}\right)\right)^{G} .
$$

2.2. Consider the localization map:

$$
\operatorname{loc}_{K}: \mathrm{H}_{\mathrm{fl}}^{1}(K, A) \longrightarrow \bigoplus_{v} \mathrm{H}_{\mathrm{fl}}^{1}\left(K_{v}, A\right) .
$$

The generalized Cassels-Tate dual exact sequence of [GT07, Main Theorem] identifies, for any integer $m$, the cokernel of $l o c_{K}$ with the Pontryagin dual of

$$
T_{m} \operatorname{Sel}\left(A^{t} / K\right):=\lim _{\longleftarrow} \operatorname{Sel}_{m^{n}}\left(A^{t} / K\right) .
$$

In our case $A$ is an elliptic curve, so $A=A^{t}$. Taking the inverse limit of the sequence

$$
0 \longrightarrow A(K) / m^{n} A(K) \longrightarrow \operatorname{Sel}_{m^{n}}(A / K) \longrightarrow \amalg(A / K)\left[m^{n}\right] \longrightarrow 0
$$

we see that $T_{m} \operatorname{Sel}(A / K)$ is always finite, and trivial for almost every $m$, because so are $A(K)$ and $\amalg(A / K)$. Hence $l o c_{K}$ has finite cokernel and, by the inclusions

$$
\mathrm{H}^{1}\left(\Gamma, A\left(L_{v_{0}}\right)\right) \subseteq \mathrm{H}_{\mathrm{fl}}^{1}\left(K_{v_{0}}, A\right) \subseteq \bigoplus_{v} \mathrm{H}_{\mathrm{fl}}^{1}\left(K_{v}, A\right),
$$

it follows that

$$
\mathcal{H}:=\mathrm{H}^{1}\left(\Gamma, A\left(L_{v_{0}}\right)\right) \cap l o c_{K}\left(\mathrm{H}_{\mathrm{fl}}^{1}(K, A)\right)
$$

has finite index in $\mathrm{H}^{1}\left(\Gamma, A\left(L_{v_{0}}\right)\right)$. Since the latter is $p$-divisible, we must have

$$
\mathcal{H}=\mathrm{H}^{1}\left(\Gamma, A\left(L_{v_{0}}\right)\right) \subseteq l o c_{K}\left(\mathrm{H}_{\mathrm{fl}}^{1}(K, A)\right) .
$$


Write $E:=\operatorname{loc}_{K}^{-1}\left(\mathrm{H}^{1}\left(\Gamma, A\left(L_{v_{0}}\right)\right)\right)$. We have an exact sequence

$$
0 \longrightarrow \amalg(A / K) \longrightarrow E \stackrel{l o c_{K}}{\longrightarrow} \mathrm{H}^{1}\left(\Gamma, A\left(L_{v_{0}}\right)\right) \longrightarrow 0 .
$$

It follows that $\operatorname{loc}_{L}\left(\operatorname{res}_{L / K}(E)\right)=0$, because restriction and localization commute and $\mathrm{H}^{1}\left(\Gamma, A\left(L_{v_{0}}\right)\right)$ has trivial image in $\oplus_{v} \mathrm{H}_{\mathrm{fl}}^{1}\left(L_{v}, A\right)$.

Let $D$ be the divisible part of $E$ : then $D \simeq \mathbb{Q}_{p} / \mathbb{Z}_{p}$, since $\amalg(A / K)$ is a finite group. By construction $D$ is a subgroup of $\mathrm{H}_{\mathrm{fl}}^{1}(K, A)$, killed by $\operatorname{loc}_{L} \circ \mathrm{res}_{L / K}$ : that is,

$$
\operatorname{res}_{L / K}(D) \subset \amalg_{p^{\infty}}(A / L)^{\Gamma} .
$$

2.3. For the proof that $X_{p}(A / L)$ is not torsion, we are going to reason by contradiction. Thus in the following we assume that $X_{p}(A / L)$ is torsion.

For each $n$, let $\amalg_{p^{\infty}}\left(A / K_{n}\right)_{d i v}$ and $\operatorname{Sel}_{p \infty}\left(A / K_{n}\right)_{d i v}$ respectively denote the $p$-divisible part of $\amalg_{p^{\infty}}\left(A / K_{n}\right)$ and $\operatorname{Sel}_{p \infty}\left(A / K_{n}\right)$. Denote

$$
\mathfrak{b}_{n}=\amalg_{p^{\infty}}\left(A / K_{n}\right) / \amalg_{p^{\infty}}\left(A / K_{n}\right)_{d i v}=\operatorname{Sel}_{p^{\infty}}\left(A / K_{n}\right) / \operatorname{Sel}_{p^{\infty}}\left(A / K_{n}\right)_{d i v} .
$$

Let $\mathfrak{r}_{m}^{n}: \mathfrak{b}_{m} \rightarrow \mathfrak{b}_{n}$ and $\mathfrak{k}_{m}^{n}: \mathfrak{b}_{n} \rightarrow \mathfrak{b}_{m}$ be the morphisms induced respectively from the restriction

and the co-restriction

$$
\mathrm{H}_{\mathrm{fl}}^{1}\left(K_{m}, A\right) \longrightarrow \mathrm{H}_{\mathrm{fl}}^{1}\left(K_{n}, A\right)
$$

$$
\mathrm{H}_{\mathrm{fl}}^{1}\left(K_{n}, A\right) \longrightarrow \mathrm{H}_{\mathrm{fl}}^{1}\left(K_{m}, A\right) .
$$

The Cassels-Tate pairing induces, for every $n$, a perfect alternating pairing:

$$
\langle,\rangle_{n}: \mathfrak{b}_{n} \times \mathfrak{b}_{n} \longrightarrow \mathbb{Q}_{p} / \mathbb{Z}_{p}
$$

Then the module $\mathfrak{b}_{\infty}:=\lim \mathfrak{b}_{m}$ is identified with the Pontryagin dual of $\mathfrak{b}:=$ $\lim _{\longleftarrow} \mathfrak{b}_{n}$. Set

$$
\begin{aligned}
\operatorname{Sel}_{d i v}(A / L) & :=\underset{n}{\lim } \operatorname{Sel}_{p^{\infty}}\left(A / K_{n}\right)_{d i v}, \\
Y_{p}\left(A / K_{n}\right) & :=\left(\operatorname{Sel}_{p^{\infty}}\left(A / K_{n}\right)_{d i v}\right)^{\vee}
\end{aligned}
$$

and

$$
Y_{p}(A / L):={\underset{\lim }{n}}_{n} Y_{p}\left(A / K_{n}\right)=\operatorname{Sel}_{d i v}(A / L)^{\vee} .
$$

Again, the notation $\bullet \vee$ denotes the Pontryagin dual of $\bullet$. We have the following short exact sequence:

$$
0 \longrightarrow \mathfrak{b} \longrightarrow X_{p}(A / L) \longrightarrow Y_{p}(A / L) \longrightarrow 0 .
$$

In particular, if $X_{p}(A / L)$ is a finitely generated torsion $\Lambda$-module, so are the modules $\mathfrak{b}$ and $Y_{p}(A / L)$.

Definition 2.3.1. An element $f \in \Lambda(\Gamma)$ is simple if there exist $\gamma \in \Gamma-\Gamma^{p}$ and $\zeta \in \boldsymbol{\mu}_{p^{\infty}}$ such that

$$
f=f_{\gamma, \zeta}:=\prod_{\sigma \in \operatorname{Gal}\left(\mathbb{Q}_{p}(\zeta) / \mathbb{Q}_{p}\right)}(\gamma-\sigma(\zeta)) .
$$

The following result was proved in [Tan12, Theorem 5],

Theorem 2.3.1. Suppose $X_{p}(A / L)$ is a torsion $\Lambda$-module. Then there exist pairwise relatively prime simple elements $f_{1}, \ldots, f_{m}(m \geq 1)$ such that

$$
f_{1} \cdots f_{m} \cdot \operatorname{Sel}_{d i v}(A / L)=0 .
$$


We will also need the following two lemmas:

Lemma 2.3.2. For $n \geq m$ the restriction map

$$
\operatorname{Sel}_{p \infty}\left(A / K_{m}\right)_{d i v} \longrightarrow\left(\operatorname{Sel}_{p} \infty\left(A / K_{n}\right)^{\Gamma^{(m)}}\right)_{d i v}
$$

is surjective.

Proof. The commutative diagram of exact sequences

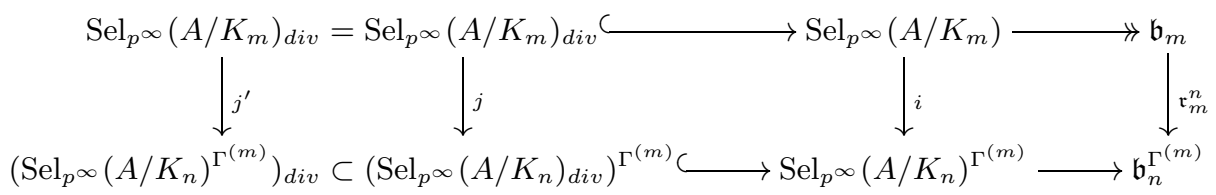

induces the exact sequence

$$
\operatorname{Ker}\left(\mathfrak{r}_{m}^{n}\right) \longrightarrow \operatorname{Coker}(j) \longrightarrow \operatorname{Coker}(i) .
$$

Since $\operatorname{Ker}\left(\mathfrak{r}_{m}^{n}\right)$ is finite while $\operatorname{Coker}\left(j^{\prime}\right)$ is $p$-divisible, it is sufficient to show that Coker $(i)$ is annihilated by some positive integer. Consider the commutative diagram of exact sequences

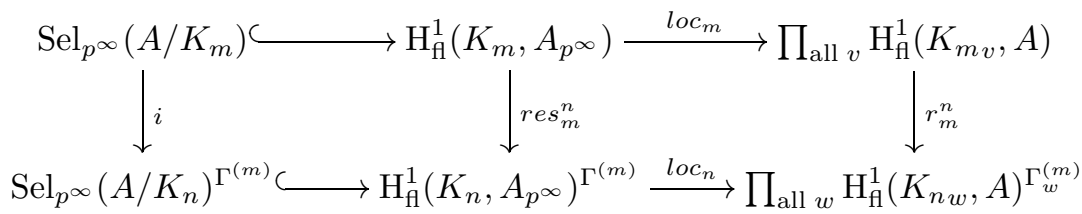

that induces the exact sequence

$$
\operatorname{Ker}\left(\operatorname{Im}\left(l o c_{m}\right) \stackrel{r_{m}^{n}}{\longrightarrow} \operatorname{Im}\left(l o c_{n}\right)\right) \longrightarrow \operatorname{Coker}(i) \longrightarrow \operatorname{Coker}\left(r e s_{m}^{n}\right) .
$$

By the Hochschild-Serre spectral sequence, the right-hand term $\operatorname{Coker}\left(\right.$ res $\left._{m}^{n}\right)$ is a subgroup of $\mathrm{H}^{2}\left(K_{n} / K_{m}, A_{p}\left(K_{n}\right)\right)$, and hence is annihilated by $p^{d(n-m)}=\left[K_{n}\right.$ : $\left.K_{m}\right]$. Similarly, the left-hand term, being a subgroup of $\prod_{v} \mathrm{H}^{1}\left(K_{n v} / K_{m v}, A\left(K_{n v}\right)\right)$, is also annihilated by $\left[K_{n}: K_{m}\right]$.

Lemma 2.3.3. If $X_{p}(A / L)$ is a torsion $\Lambda$-module, then there exists some $N$ such that

$$
\operatorname{Sel}_{p^{\infty}}\left(A / K_{n}\right)_{d i v}=\left(\operatorname{Sel}_{p^{\infty}}\left(A / K_{n}\right)_{d i v}\right)^{\Gamma^{(m)}}=\left(\operatorname{Sel}_{p^{\infty}}\left(A / K_{n}\right)^{\Gamma^{(m)}}\right)_{d i v}
$$

holds for all $n \geq m \geq N$.

Proof. The second equality is an easy consequence of the first one. The assumption $\Gamma=\gamma^{\mathbb{Z}_{p}}$ implies that if $f \in \Lambda$ is simple, then $f$ divides $\gamma^{p^{m}}-1$ for some $m$. Therefore, by Theorem 2.3.1, there exists an integer $N$ such that $\left(\gamma^{p^{N}}-1\right) \operatorname{Sel}_{d i v}(A / L)^{\Gamma^{(n)}}=0$ for every $n$. The kernel of the map $\operatorname{Sel}_{p^{\infty}}\left(A / K_{n}\right)_{d i v} \rightarrow \operatorname{Sel}_{d i v}(A / L)^{\Gamma^{(n)}}$ is finite since $A_{p^{\infty}}(L)$ is finite. This implies that $\left(\gamma^{p^{N}}-1\right) \operatorname{Sel}_{p^{\infty}}\left(A / K_{n}\right)$ div must be trivial, because it is both finite and $p$-divisible.

Proposition 2.3.4. The $\mathbb{Z}_{p}$-rank of $\mathfrak{b} / T \mathfrak{b}$ equals 1 . 
Proof. First, note that, by Lemma 2.3.2, the finiteness of $\operatorname{Sel}_{p}(A / K)$ implies $\operatorname{Sel}_{p \infty}\left(A / K_{n}\right)^{\Gamma}$ is finite for every $n \geq 0$. Then Lemma 2.3.2 and Lemma 2.3.3 imply that for $n \gg 0$,

$$
\operatorname{Sel}_{d i v}(A / L)^{\Gamma}=\left(\operatorname{Sel}_{d i v}(A / L)^{\Gamma^{(n)}}\right)^{\Gamma}=\left(\operatorname{Sel}_{p^{\infty}}\left(A / K_{n}\right)_{d i v}\right)^{\Gamma} \subset \operatorname{Sel}_{p^{\infty}}\left(A / K_{n}\right)^{\Gamma}
$$

is also finite. By duality, the $\mathbb{Z}_{p}$-module $Y_{p}(A / L) / T Y_{p}(A / L)$ is finite. This means the characteristic ideal of $Y_{p}(A / L)$ is relatively prime to $(T)$. Hence, there is some $\xi \in \Lambda$, relatively prime to $T$ such that $\xi \cdot Y_{p}(A / L)$ is pseudo-null (finite). Replacing $\xi$ by some suitable $p^{m} \xi$, we can assert that $\xi \cdot Y_{p}(A / L)=0$. Then $Y_{p}(A / L)[T]$, being annihilated by $T$ and $\xi$, is also finite (hence trivial, because $Y_{p}(A / L)$, as a submodule of the $\mathbb{Z}_{p}$-free part of $X_{p}(A / L)$, is a free $\mathbb{Z}_{p}$-module of finite rank). Since (10) and the snake lemma yield the exact sequence

$$
Y_{p}(A / L)[T] \longrightarrow \mathfrak{b} / T \mathfrak{b} \longrightarrow X_{p}(A / L) / T X_{p}(A / L) \longrightarrow Y_{p}(A / L) / T Y_{p}(A / L),
$$

it follows from Corollary 2.1.2 that the $\mathbb{Z}_{p}$-rank of $\mathfrak{b} / T \mathfrak{b}$ is at most 1 .

For the other inequality, consider the composition

$$
\pi: D \stackrel{r e s_{L / K}}{\longrightarrow} \amalg_{p^{\infty}}(A / L)^{\Gamma} \longrightarrow \mathfrak{b}_{\infty}^{\Gamma} .
$$

Let $\circledast$ denote the preimage of $D$ under the natural surjection from $\mathrm{H}^{1}\left(K, A_{p}\right)$ to the $p$-primary part of $\mathrm{H}^{1}(K, A)$. Because $A(K)$ is finite, the exact sequence

$$
0 \longrightarrow \mathbb{Q}_{p} / \mathbb{Z}_{p} \otimes A(K) \longrightarrow \mathbb{\longrightarrow} \longrightarrow 0
$$

implies that $Æ \simeq D$ is of corank 1 over $\mathbb{Z}_{p}$. It follows that $\operatorname{Res}_{L / K}(Æ) \subset \operatorname{Sel}_{p}(A / L)^{\Gamma}$ is also of corank 1 over $\mathbb{Z}_{p}$, since $\operatorname{Ker}\left(\operatorname{Res}_{L / K}\right)=\mathrm{H}^{1}\left(\Gamma, A_{p^{\infty}}(L)\right)$ is of finite order, by Corollary 2.0.3. If the image of $\pi$ were finite (and thus trivial, since $D$ is $p$ divisible), then $\operatorname{Res}_{L / K}(Æ)$ would be contained in $\operatorname{Sel}_{\text {div }}(A / L)^{\Gamma}$, which has just been shown to be finite. This is absurd. Therefore, the corank of $\mathfrak{b}_{\infty}^{\Gamma}$ is at least 1 , and the desired inequality followed by the duality.

Proposition 2.3 .4 implies that there exist some $r \geq 1$ and $\xi_{i} \in \Lambda, i=1, \ldots, s$, coprime to $T$ such that $\mathfrak{b}$ is pseudo-isomorphic to $[\mathfrak{b}]:=\Lambda / T^{r} \Lambda \oplus \bigoplus_{i}^{s} \Lambda / \xi_{i} \Lambda$. Since $[\mathfrak{b}]$ contains no non-trivial pseudo-null submodule, we actually have a short exact sequence

$$
0 \longrightarrow \Lambda / T^{r} \Lambda \oplus \bigoplus_{i}^{s} \Lambda / \xi_{i} \Lambda \longrightarrow \mathfrak{b} \longrightarrow N \longrightarrow 0,
$$

with $N$ pseudo-null. For each $n$, let $\mathfrak{r}_{n}: \mathfrak{b}_{n} \rightarrow \mathfrak{b}_{\infty}$ and $\mathfrak{k}_{n}: \mathfrak{b} \rightarrow \mathfrak{n}_{n}$ be the natural maps associated to the injective and projective limits. They are dual to each other.

Lemma 2.3.5. The kernel of $\mathfrak{r}_{n}$ and the cokernel of $\mathfrak{k}_{n}$ are of bounded orders as $n$ varies.

Proof. Suppose the natural projection $\operatorname{Sel}_{p \infty}\left(A / K_{n}\right) \longrightarrow \mathfrak{b}_{n}$ sends each $x$ to $\bar{x}$. Assume that $\bar{x} \in \operatorname{Ker}\left(\mathfrak{r}_{n}\right)$. Then $\operatorname{Res}_{L / K_{n}}(x) \in \operatorname{Sel}_{d i v}(A / L)$. Consider the restriction map

$$
\mathrm{R}_{n}: \operatorname{Sel}_{p^{\infty}}\left(A / K_{n}\right)_{d i v} \longrightarrow \operatorname{Sel}_{d i v}(A / L) .
$$

Since $\operatorname{Sel}_{p}(A / L)$ is cotorsion over $\Lambda$, the divisible subgroup $\operatorname{Sel}_{d i v}(A / L)$ must be cofinitely generated over $\mathbb{Z}_{p}$. Thus for $n$ sufficiently large, $\mathrm{R}_{n}$ is surjective. For such $n$, there exists $y \in \operatorname{Sel}_{p^{\infty}}\left(A / K_{n}\right)_{d i v}$ such that $\operatorname{Res}_{L / K_{n}}(y)=\operatorname{Res}_{L / K_{n}}(x)$. 
Since $\overline{x-y}=\bar{x}$ and $x-y$ is contained in $\operatorname{Ker}\left(\operatorname{Res}_{L / K_{n}}(x)\right)=\mathrm{H}^{1}\left(\Gamma^{(n)}, A_{p}(L)\right)$, by Corollary 2.0.3, the order of $\operatorname{Ker}\left(\mathfrak{r}_{n}\right)$ is bounded.

Lemma 2.3.6. Let $r$ be as in (12). Then the indexes of the subgroups $\mathfrak{k}_{n}\left(\mathfrak{b}\left[T^{r}\right]\right) \subset$ $\mathfrak{b}_{n}\left[T^{r}\right]$ are bounded as $n$ varies.

Proof. Lemma 2.3.5 implies that $\mathfrak{b}_{n}\left[T^{r}\right] / \mathfrak{k}_{n}(\mathfrak{b}) \cap \mathfrak{b}_{n}\left[T^{r}\right]$, embedded as a subgroup of $\mathfrak{b}_{n} / \mathfrak{k}_{n}(\mathfrak{b})$, is of bounded order. It is sufficient to show that $\mathfrak{e}_{n}:=\mathfrak{k}_{n}(\mathfrak{b}) \cap$ $\mathfrak{b}_{n}\left[T^{r}\right] / \mathfrak{k}_{n}\left(\mathfrak{b}\left[T^{r}\right]\right)$ is also of bounded order. Denote $\mathfrak{f}_{n}:=\mathfrak{k}_{n}(\mathfrak{b}) / \mathfrak{k}_{n}\left(\mathfrak{b}\left[T^{r}\right]\right)$. Then $\mathfrak{e}_{n} \subset \mathfrak{f}_{n}\left[T^{r}\right]$. Suppose the pseudo-null $N$ in (12) is annihilated by some $\eta$ coprime to $T$. Then $g=\eta \cdot \xi_{1} \cdots \cdots \xi_{s}$ is also coprime to $T$ and we have $g \cdot \mathfrak{b} \in[\mathfrak{b}]\left[T^{r}\right] \subset \mathfrak{b}\left[T^{r}\right]$. This implies $g \cdot \mathfrak{f}_{n}=0$, and hence $\mathfrak{e}_{n}$ is annihilated by some $p$-power $p^{c} \in\left(T^{r}, g\right) \subset \Lambda$. Since $\mathfrak{b}$ is $\Lambda$-torsion, it is a finitely generated $\mathbb{Z}_{p}$-module. We have the composition of surjective homomorphisms $\mathbb{Z}_{p}^{l} \rightarrow \mathfrak{b} \rightarrow \mathfrak{k}_{n}(\mathfrak{b}) \rightarrow \mathfrak{f}_{n}$ for some $l$. Then $\varrho^{-1}\left(\mathfrak{e}_{n}\right)$ is a free $\mathbb{Z}_{p}$-module of rank at most $l$ and we have a surjective homomorphism $\varrho^{-1}\left(\mathfrak{e}_{n}\right) \rightarrow \mathfrak{e}_{n}$. Therefore, $\mathfrak{e}_{n}$ can be generated by a subset of cardinality at most $l$. Hence its order is bounded by $p^{l c}$.

2.4. In order to exploit the anticyclotomic assumption, we now consider the action of $G:=\operatorname{Gal}(K / k)=\langle\tau\rangle$ on $\mathfrak{b}$. We lift $G$ to a subgroup of $\operatorname{Gal}(L / k)$. Note that the maps $l o c_{K}$ of (8) and $\pi$ of (11) are both compatible with the action of $G$.

Lemma 2.4.1. There exists $x \in \mathfrak{b}\left[T^{r}\right]^{G}$ such that $\left[\mathfrak{b}\left[T^{r}\right]: \Lambda x\right]<\infty$.

Proof. Remark 2.1 .3 yields that $(1-\tau) D$ is contained in $\operatorname{Ker}\left(\operatorname{loc}_{K}\right)=\amalg(A / K)$ and hence is trivial (because $D$ is divisible). Thus we have $\pi(D)=\pi\left(D_{G}\right)=\pi(D)_{G}$ and by duality (see the proof of Proposition 2.3.4) it follows that $(\mathfrak{b} / T \mathfrak{b})^{G}$ has rank 1 over $\mathbb{Z}_{p}$. Also the image of $\mathfrak{b}\left[T^{r}\right]$ in $\mathfrak{b} / T \mathfrak{b}$ is of $\mathbb{Z}_{p}$-rank 1 , by (12), so there must be $y \in \mathfrak{b}\left[T^{r}\right]$ such that $y \bmod T \mathfrak{b}$ is $G$-invariant and has infinite order. Choose $x:=(1+\tau) y$. Then $x \in \mathfrak{b}\left[T^{r}\right]^{G}$ and $\Lambda x$ has finite index in $\mathfrak{b}\left[T^{r}\right] \sim \Lambda / T^{r} \Lambda$ since $x \equiv 2 y(\bmod T \mathfrak{b})$ generates a free $\mathbb{Z}_{p}$-module in $\mathfrak{b} / T \mathfrak{b}$.

Let $\mathfrak{c}:=\Lambda x$ and $\mathfrak{c}_{n}:=\mathfrak{k}_{n}(\mathfrak{c})$.

Lemma 2.4.2. As $n$ varies, the orders of the cokernels of the maps $\mathfrak{c}_{n} \rightarrow \mathfrak{b}_{n}\left[T^{r}\right]$ and $\mathfrak{c}_{n} \rightarrow \mathfrak{b}_{n} \rightarrow \mathfrak{b}_{n} / T^{r} \mathfrak{b}_{n}$ are bounded.

Proof. By (12) the cokernel of $\mathfrak{b}\left[T^{r}\right] \rightarrow \mathfrak{b} \rightarrow \mathfrak{b} / T^{r} \mathfrak{b}$ is finite. Then apply Lemma 2.3.6 and Lemma 2.4.1.

Lemma 2.4.3. We have

$$
\left\langle\mathfrak{c}_{n}, \mathfrak{c}_{n}\right\rangle_{n} \subset\left(\mathbb{Q}_{p} / \mathbb{Z}_{p}\right)[2] .
$$

So $\left\langle\mathfrak{c}_{n}, \mathfrak{c}_{n}\right\rangle_{n}$ has at most 2 elements and it is trivial if $p \neq 2$. Let $\#: \Lambda \rightarrow \Lambda$ denote the $\mathbb{Z}_{p}$-algebra isomorphism induced by $\gamma \mapsto \gamma^{-1}, \gamma \in \Gamma$.

Proof. Write $x_{n}:=\mathfrak{k}_{n}(x)$. For any $\lambda \in \Lambda$ we have

$$
\left\langle x_{n}, \lambda x_{n}\right\rangle_{n}=-\left\langle\lambda x_{n}, x_{n}\right\rangle_{n}=-\left\langle x_{n}, \lambda^{\#} x_{n}\right\rangle_{n},
$$

using first the fact that $\langle,\rangle_{n}$ is alternating and then its $\Gamma$-equivariance. On the other hand, the pairing is also $G$-invariant and $x=\tau x$ implies

$$
\left\langle x_{n}, \lambda x_{n}\right\rangle_{n}=\left\langle\tau x_{n}, \tau\left(\lambda x_{n}\right)\right\rangle_{n}=\left\langle x_{n}, \lambda^{\#} x_{n}\right\rangle_{n},
$$


because $\tau(\lambda x)=\left(\tau \lambda \tau^{-1}\right) \tau x$ and the action of $\tau$ on $\Lambda$ is precisely $\lambda \mapsto \lambda^{\#}$, by the anticyclotomic hypothesis. Equalities (13) and (14) together prove

$$
2\left\langle x_{n}, \lambda^{\#} x_{n}\right\rangle_{n}=0
$$

and this suffices, since $\mathfrak{c}_{n}=\Lambda x_{n}$.

2.5. Now we can finally obtain a contradiction. The Cassels-Tate pairing makes $\mathfrak{b}_{n}\left[T^{r}\right]$ and $\mathfrak{b}_{n} / T^{r} \mathfrak{b}_{n}$ dual to each other. Lemma 2.4 .2 implies that the subgroup

$$
\left\langle\mathfrak{c}_{n}, \mathfrak{c}_{n}\right\rangle_{n} \subseteq\left\langle\mathfrak{b}_{n}\left[T^{r}\right], \mathfrak{b}_{n} / T^{r} \mathfrak{b}_{n}\right\rangle_{n}
$$

has bounded index as $n$ varies. Since $\pi(D) \subseteq \mathfrak{b}_{\infty}[T] \subseteq \mathfrak{b}_{\infty}\left[T^{r}\right]=\lim _{n} \mathfrak{b}_{n}\left[T^{r}\right]$ is infinite, we must have

$$
\bigcup_{n}\left\langle\mathfrak{c}_{n}, \mathfrak{c}_{n}\right\rangle_{n}=\mathbb{Q}_{p} / \mathbb{Z}_{p}
$$

a contradiction to Lemma 2.4.3.

\section{The AnALYTiC SIDE}

In this example $X_{p}(A / L)$ is non-torsion, whence its characteristic ideal is trivial. In the spirit of the Iwasawa Main Conjecture one expects that the corresponding $p$-adic $L$-function should be 0 . Here we verify this.

In the function field setting, non-isotrivial elliptic curves are known to be modular: that is, there is a cuspidal automorphic function $f$ associated with $A$; its level is $\mathfrak{n}$, the conductor of $A / K$ (as a divisor of $K$ ). For any $n \geq 0$, let $\mathfrak{d}_{n}$ denote the divisor $n v_{0}$ and let $K\left(\mathfrak{d}_{n}\right) / K$ be the corresponding ray class field. It is shown in Tan93. how to construct a modular element $\Theta_{\mathfrak{d}_{n}, f} \in \mathbb{Z}_{p}\left[\operatorname{Gal}\left(K\left(\mathfrak{d}_{n}\right) / K\right)\right]$, such that for each $\omega \in \operatorname{Gal}\left(K\left(\mathfrak{d}_{n}\right) / K\right)^{\vee}$ one has

$$
\omega\left(\Theta_{\mathfrak{d}_{n}, f}\right)=\tau_{\omega} \cdot L(A, \omega, 1),
$$

where $\tau_{\omega}$ is a Gauss sum.

By [Tan93, Proposition 2, 2.(d)], the maps

$$
\mathbb{Z}_{p}\left[\operatorname{Gal}\left(K\left(\mathfrak{d}_{n+1}\right) / K\right)\right] \longrightarrow \mathbb{Z}_{p}\left[\operatorname{Gal}\left(K\left(\mathfrak{d}_{n}\right) / K\right)\right]
$$

send the modular elements $\Theta_{\mathfrak{d}_{n}, f}$ into each other, so that one can take their limit $\tilde{\Theta}$. Any abelian extension of $K$ totally ramified above $v_{0}$ and unramified elsewhere is contained in $\cup K\left(\mathfrak{d}_{n}\right)$ : in particular this holds for our $L$. Let $\Theta$ be the image of $\tilde{\Theta}$ under the projection

$$
\underset{\lim }{\longleftarrow} \mathbb{Z}_{p}\left[\operatorname{Gal}\left(K\left(\mathfrak{d}_{n}\right) / K\right)\right] \longrightarrow \Lambda \text {. }
$$

Equation (15) shows that $\Theta$ satisfies the interpolation property required for the $p$-adic $L$-function. Observe that $\Theta_{\mathfrak{d}_{n}, f}$ is invariant under the action of $\operatorname{Gal}(K / k)$, since the modular elements are already defined above $k$. Thus, as elements of $\mathbb{Z}_{p}[[\operatorname{Gal}(L / k)]]$,

$$
\Theta=\tau \cdot \Theta \cdot \tau^{-1}=\Theta^{\#}
$$

On the other hand, by [Tan93, Proposition 3] we have

$$
\Theta_{\mathfrak{d}_{n}, f}=-\Theta_{\mathfrak{d}_{n}, f}^{\#} \cdot \eta
$$

where $\eta \in \operatorname{Gal}\left(K\left(\mathfrak{d}_{n}\right) / K\right)$ corresponds to the divisor $\mathfrak{n}^{\prime}=\mathfrak{n}-v_{0}$. This implies that $\Theta=0$. 


\section{REFERENCES}

[GT07] Cristian D. González-Avilés and Ki-Seng Tan, A generalization of the CasselsTate dual exact sequence, Math. Res. Lett. 14 (2007), no. 2, 295-302, DOI 10.4310/MRL.2007.v14.n2.a11. MR.2318626 (2008k:11067)

[Lan91] William E. Lang, Extremal rational elliptic surfaces in characteristic $p$. I. Beauville surfaces, Math. Z. 207 (1991), no. 3, 429-437, DOI 10.1007/BF02571400. MR.1115175 (92f:14032)

[Maz72] Barry Mazur, Rational points of abelian varieties with values in towers of number fields, Invent. Math. 18 (1972), 183-266. MR0444670 (56 \#3020)

[Mil75] J. S. Milne, On a conjecture of Artin and Tate, Ann. of Math. (2) 102 (1975), no. 3, 517-533. MR0414558(54 \#2659)

[Mil80] James S. Milne, Étale cohomology, Princeton Mathematical Series, vol. 33, Princeton University Press, Princeton, N.J., 1980. MR559531 (81j:14002)

[Mil86a] J. S. Milne, Arithmetic duality theorems, Perspectives in Mathematics, vol. 1, Academic Press Inc., Boston, MA, 1986. MR881804(88e:14028)

[Shi92] Tetsuji Shioda, Some remarks on elliptic curves over function fields, Astérisque 209 (1992), 12, 99-114. Journées Arithmétiques, 1991 (Geneva). MR1211006 (94d:11046)

[SU13] Christopher Skinner and Eric Urban, The Iwasawa Main Conjectures for $G L_{2}$, Invent. Math. 195 (2014), no. 1, 1-277, DOI 10.1007/s00222-013-0448-1. MR3148103

[Tan93] Ki-Seng Tan, Modular elements over function fields, J. Number Theory 45 (1993), no. 3, 295-311, DOI 10.1006/jnth.1993.1079. MR.1247386 (95d:11158)

[Tan12] Ki-Seng Tan, Selmer groups over $\mathbb{Z}_{p}^{d}$-extensions, Math. Ann. 359 (2014), no. 3-4, 10251075, DOI 10.1007/s00208-014-1023-9. MR.3231024

[Ta66] John Tate, On the conjectures of Birch and Swinnerton-Dyer and a geometric analog, Séminaire Bourbaki, Vol. 9, Soc. Math. France, Paris, 1995, pp. Exp. No. 306, 415-440. MR.1610977

School of Mathematical Sciences, Capital Normal University, Beijing 100048, PeoPLE's RePUblic of ChinA

E-mail address: kinglaihonkon@gmail.com

Department of Mathematics, National Taiwan University. Taipei 10764, Taiwan

Current address: Department of Mathematical Sciences, Xi'an Jiaotong-Liverpool University, No. 111 Ren'ai Road, Dushu Lake Higher Education Town, Suzhou Industrial Park, Suzhou 215123 Jiangsu, People's Republic of China.

E-mail address: longhi@math.ntu.edu.tw

Department of Mathematics, National Taiwan University, Taipei 10764, Taiwan

E-mail address: tan@math.ntu.edu.tw

College of Engineering, Mathematics and Physical Sciences, University of Exeter, North Park Road, Exeter, United Kingdom

Current address: Department of Information and Communication Sciences, Faculty of Science and Technology, Sophia University, 4 Yonbancho, Chiyoda-ku, Tokyo 102-0081 Japan

E-mail address: f-trihan-52m@sophia.ac.jp 